\title{
Nueva localidad para Avellanita bustillosii Phil. (Euphorbiaceae) en la Región de O’Higgins y su estado de conservación
}

\section{New location to Avellanita bustillosii Phil. (Euphorbiaceae) in O'Higgins Region and its conservation status}

\author{
Patricio Medina ${ }^{1 *}$ \& Gloria Rojas ${ }^{2}$ \\ 1 Investigador independiente, Laguna San Rafael 3117, Puerto Varas, Chile. \\ ${ }^{2}$ Museo Nacional Historia Natural, Sección Botánica, casilla 787, Santiago, Chile. \\ *alstroemeds@gmail.com
}

\begin{abstract}
A new record for Avellanita bustillosii Phil. is added. Botanical surveys in pre-andean mountain range of O'Higgins Region leads to find a new population. Their distributional southern limit is extended about $26.5 \mathrm{~km}$. This population was found between 700 and 880 masl, in the northern slope of the Retamal foothill and the slopes with eastern exposure of the Trocalán hill, both located at $11 \mathrm{~km}$ east of the town of Requinoa, Cachapoal Province, O'Higgins Region. This finding it's a contribution to the taxon chorology and reveals the lack of information available about their distributional territory. This is the first population described to Central Chile pre-andean mountain range. A re-evaluation to its conservation status is provided.
\end{abstract}

Avellanita bustillosii Phil., la "avellanita", elemento florístico de presencia ocasional que compone el sotobosque de comunidades de bosques esclerófilos de la Cordillera de la Costa de la Región Metropolitana y de la Región de O'Higgins (Hechenleitner et al. 2005, Serra et al. 2005, Ricci \& Benoit 2008, Medina 2010).

La especie conforma parte de la lista de especies con escasa representación en áreas protegidas, pues ninguna de las poblaciones conocidas se encuentran bajo el amparo del Sistema Nacional de Áreas Silvestres protegidas del Estado (CONAMA 2004, Hechenleitner et al. 2005, CONAMA 2008, Ricci \& Benoit 2008, Medina 2010, CONAF 2017). Todas las poblaciones conocidas son de reducido tamaño, están insertas en comunidades vegetales fragmentadas, alteradas y amenazadas por diversos tipos de intervención antrópica (Videla 2008, Serra et al. 2005, Ricci \& Benoit 2008, Hechenleitner et al. 2005, Medina 2010). La población de Marga-Marga se considera extinta (Hechenleitner et al. 2005, Serra et al. 2005, Ricci \& Benoit 2008, Medina 2010).

Exploraciones efectuadas por G. Rojas (SGO 166375, 166376) en la Región de O’Higgins, revelaron la presencia de una nueva población en los faldeos de la precordillera andina que colindan con las comunas de Requínoa y Machalí. El objetivo de este trabajo consiste en dar a conocer la nueva localidad registrada para Avellanita bustillosii, junto con una breve descripción de su hábitat. Además, se amplía su límite sur de distribución.
La nueva población fue registrada tras efectuar exploraciones botánicas sucesivas durante los años 20122013 en las inmediaciones de cordones montañosos de la precordillera andina y el sendero de Chile de la comuna de Requínoa, Provincia de Cachapoal, Región de O'Higgins (Fig. 1). El área se ubica aproximadamente a $13 \mathrm{~km}$ sureste de Rancagua y $11 \mathrm{~km}$ hacia el este de la ciudad de Requínoa, $50 \mathrm{~km}$ al sureste de laguna de Aculeo (RM). Los sitios de observación se emplazan en el piedemonte con exposición norte del cerro Retamal (1.287 m s.n.m., 34 ${ }^{\circ} 18^{\prime}$ S; $70^{\circ} 42^{\prime} \mathrm{W}$ ) y en los faldeos con exposición oriente del cerro Trocalán (1.229 m s.n.m., 34¹6'S; 7041'W). Se efectuó una búsqueda intensiva en el área mediante recorridos pedestres. El rastreo fue dirigido a condiciones de hábitat similares a los observados en Aculeo (Venegas \& Contreras 2003, Ricci \& Benoit 2008) y Lo Chancón (Serra et al. 2005, Medina 2010). Se consultó material depositado en Herbario del Museo Nacional de Historia Natural (SGO) y Herbario de la Facultad de Ciencias Forestales y Conservación de la Naturaleza, Universidad de Chile (EIF). El material recolectado fue depositado en SGO.

La extensión de la presencia y el área de ocupación fueron estimadas sobre la base de criterios UICN (2012). El área de ocupación parcial de Lo Chancón se extrajo de Medina (2010). La superficie de la localidad de Aculeo fue estimada a partir de los datos de registros de los fragmentos de población proporcionados en Venegas \& Contreras 
(2003), Ricci \& Benoit (2008) y puntos de distribución expuestos por Muñoz (2009). Para estimar la extensión de la presencia se adicionó la información de distribución de la población extinta de Fundo Los Perales (Región de Valparaíso). Ambas variables fueron estimadas en software SIG de uso libre QGIS 2.18, sobre la base de literatura, informes técnicos y datos levantados por los autores en campo. El mapa de distribución se confeccionó en QGIS 2.18 (Fig. 1).

Material examinado: CHILE, Región de Valparaíso, Prov. Marga-Marga, Quilpué, Fundo los Perales (Marga-Marga), loma que separa la quebrada Ingenio de la Quebrada Los

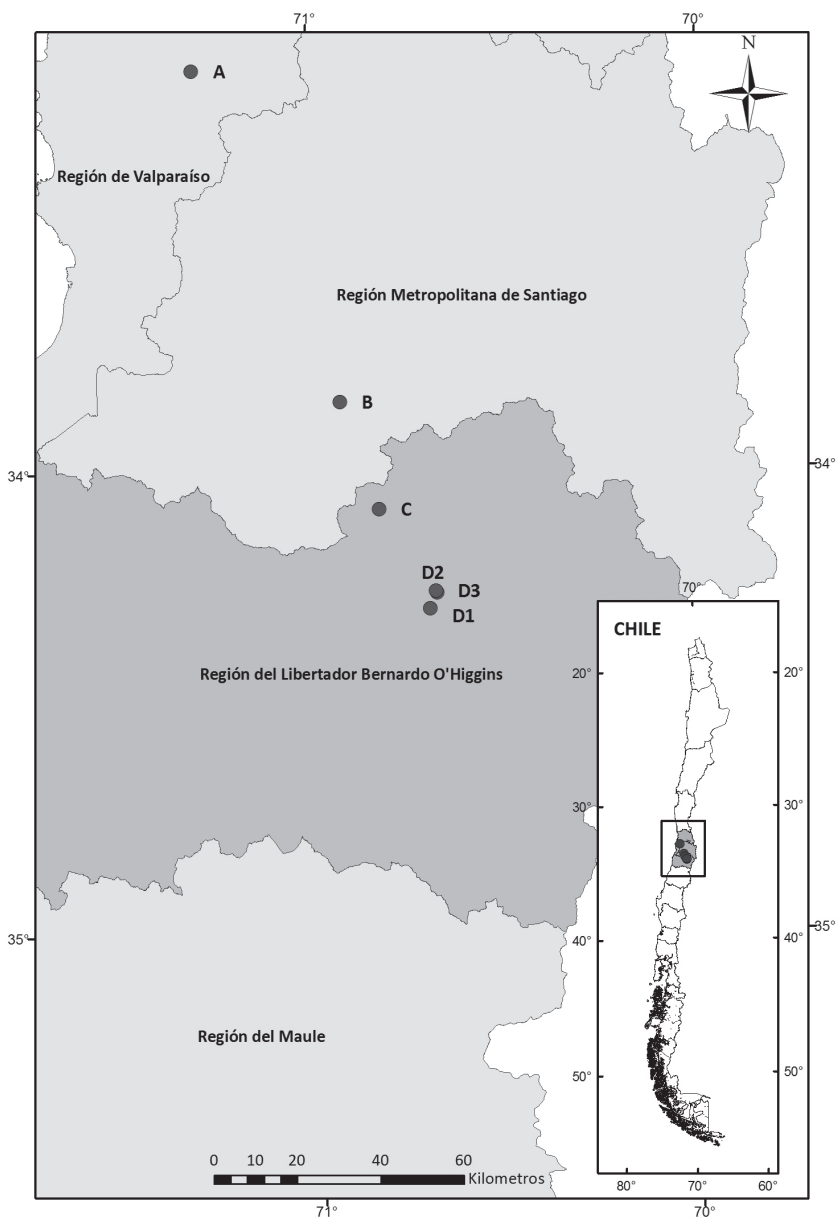

FIGURA 1: Nuevo patrón de distribución de Avellanita bustillosii en Chile. A: Límite norte de distribución (sin registros actuales), Región de Valparaíso (Jaffuel, s/n); B: Límite norte, Región Metropolitana (Medina, 1007, 1008); C: Antiguo límite sur, Región de O'Higgins (Medina, 1005, 1006) y D: Nuevo límite sur, Región de O'Higgins (Medina, 2511, 2512; Rojas, 210720 12-21). / New distributional pattern of Avellanita bustillosii in Chile. A: Past northern limit (actually without records), Valparaiso Region (Jaffuel, s/n); B: Northern limit, Metropolitan Region ((Medina, 1007, 1008); C: Old Southern record, O'Higgins Region (Medina, 1005, 1006) y D: New Southern limit, O'Higgins Region (Medina, 2511, 2512; Rojas, 210720 12-21).
Leones, $33^{\circ} 08^{\prime} \mathrm{S}, 71^{\circ} 19^{\prime} \mathrm{W}, 340 \mathrm{~m}$, IX-1910, Jaffuel s/n (SGO 51523). Región Metropolitana de Santiago, Prov.

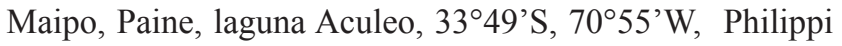
R.A. s/n (SGO 51525); laguna Aculeo,19-XI-1989, Macaya y Barriga s/n (SGO 112524); laguna Aculeo sector Los Hornos, 3351'16”'S, 7055'36”'W, 382 m, 18-V2012, Medina 1007, 1008 (SGO 161143, 161146); Cerro Cantillana, La Guachera, entrando al predio de Sr. Juan Noé, 3357'S, 7057'W, 500 m, 30-X-1994, Villagrán y Maldonado 8442 (SGO 134684). Región de O'Higgins, Prov. Cachapoal, Rancagua, Lo Chancón, 3406's, 7051'W, 520 m, 31-VIII-2003, Gamboa y Del Campo s/n (EIF 9465); 3405'11'S, 7049'46”'W, 507 m, 18-V-2012,

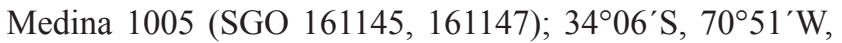
520 m, 18-XI-2004, Serra, Ricci y Luebert 2195a (SGO 152452). Prov. Cachapoal, Requínoa, piedemonte de la vertiente norte del cerro Retamal, 34 ${ }^{\circ} 18^{\prime} 13^{\prime \prime} \mathrm{S}, 70^{\circ} 42^{\prime} 02^{\prime \prime} \mathrm{W}$, 826 m, 30-XI-2013, Medina 2511, 2512 (SGO 166380, 166379); quebrada en la vertiente norte del cerro Retamal, 34¹8'14'S, 7042'10'W, 877 m, 05-XII-2013, Rojas s/n (SGO 166378); faldeos con exposición oriente del cerro Trocalán, 34¹6'07'S, 7041'03”'W, 708 m, 21-VII-2012, Rojas 210720 12-21 (SGO 166375); Localidad indefinida, 01-I-1863, Bustillos s/n (SGO 51526); 01-III-1863, Bustillos s/n (SGO 40784).

Avellanita bustillosii Phil., Linnaea 33: 238. 1864.

Nombre vernáculo: Avellanita, nogal del monte (Philippi 1895, Hechenleitner et al. 2005, Serra et al. 2005).

Distribución GeOgráfica: Se distribuye entre la comuna de Paine, Provincia de Maipo, Región Metropolitana de Santiago, hasta la comuna de Rancagua, Provincia de Cachapoal en la Región de O'Higgins (Serra et al. 2005, Ricci \& Benoit 2008, Medina 2010). La especie posee registros históricos en la Región de Valparaíso (Jaffuel \& Pirion 1921), aunque en la actualidad se considera extinta en el área (Hechenleitner et al. 2005, Serra et al. 2005, Ricci \& Benoit 2008). El presente hallazgo extiende el límite sur de distribución hasta la comuna de Requínoa, Provincia de Cachapoal, Región de O’Higgins (Fig. 1).

Descripción DEL AMBIENTE: A. bustillosii es elemento florístico ocasional, gregario, que conforma colonias localizadas en el área descrita. Cohabita en comunidades de bosque esclerófilo cuyas especies estructurantes corresponden a los fanerófitos: Peumus boldus Molina, Lithraea caustica (Molina) Hook. \& Arn., Quillaja saponaria Molina y Cryptocarya alba (Molina) Looser. En segundo orden acompañan los nanofanerófitos Retanilla trinervis (Gillies \& Hook.) Hook. \& Arn., Cestrum parqui L'Hèr., Podanthus mitiqui Lindl. y la hemicriptófita Adiantum sulphureum Kaulf. Además de la presencia de las hierbas terófitas: Anthriscus caucalis M. Bieb., Avena 
barbata Pott ex Link, Bromus berteroanus Colla y Vulpia myuros (L.) C.C. Gmel. Se registraron cuatro fragmentos de comunidades de bosque esclerófilo con presencia de avellanita. Estos se encuentran segregados espacialmente, dentro de un rango de elevación que fluctúa entre 700 y 880 m s n m., con distintas condiciones microambientales en cuanto a fisiografía, composición de especies y cubrimiento del piso.

ESTADO DE CONSERVACIÓN: Se encuentra protegida por ley sobre la base de la oficialización del DS $N^{\circ}$ 151/2007 (CONAMA 2006), bajo la categoría En Peligro y Rara (B1ab (ii) + B2ab (iii)), dado, la extrema escasez de individuos en cada población, numerosas amenazas presentes en su hábitat (Serey et al., 2007), distribución actual disyunta y eventual extinción de la población del Valle de Marga-Marga (Serra et al. 2005, Serey et al.
2007, Ricci \& Benoit 2008). Hechenleitner et al. (2005) evalúan su estatus y la clasifican en la categoría En Peligro Crítico (CR B2ab (iii)) para la Región Metropolitana y Región de O'Higgins, argumentando la existencia de la especie en sólo dos localidades sujetas a degradación. Las comunidades de bosque esclerófilo descritas en la nueva localidad están expuestas a diversas amenazas de índole antrópico: incendios forestales, extracción de tierra de hojas, pisoteo-ramoneo por ganado y diversas actividades generadas por minería a gran escala (ensanchamiento de caminos, construcción de obras de ingeniería, también existe la presencia en la cercanía de un tranque de relave minero).

La presente adición de una nueva localidad, presencia de diversos tipos de amenaza, carencia de una estimación cuantificada y actualizada del tamaño poblacional, así como del área de ocupación y extensión de la presencia,
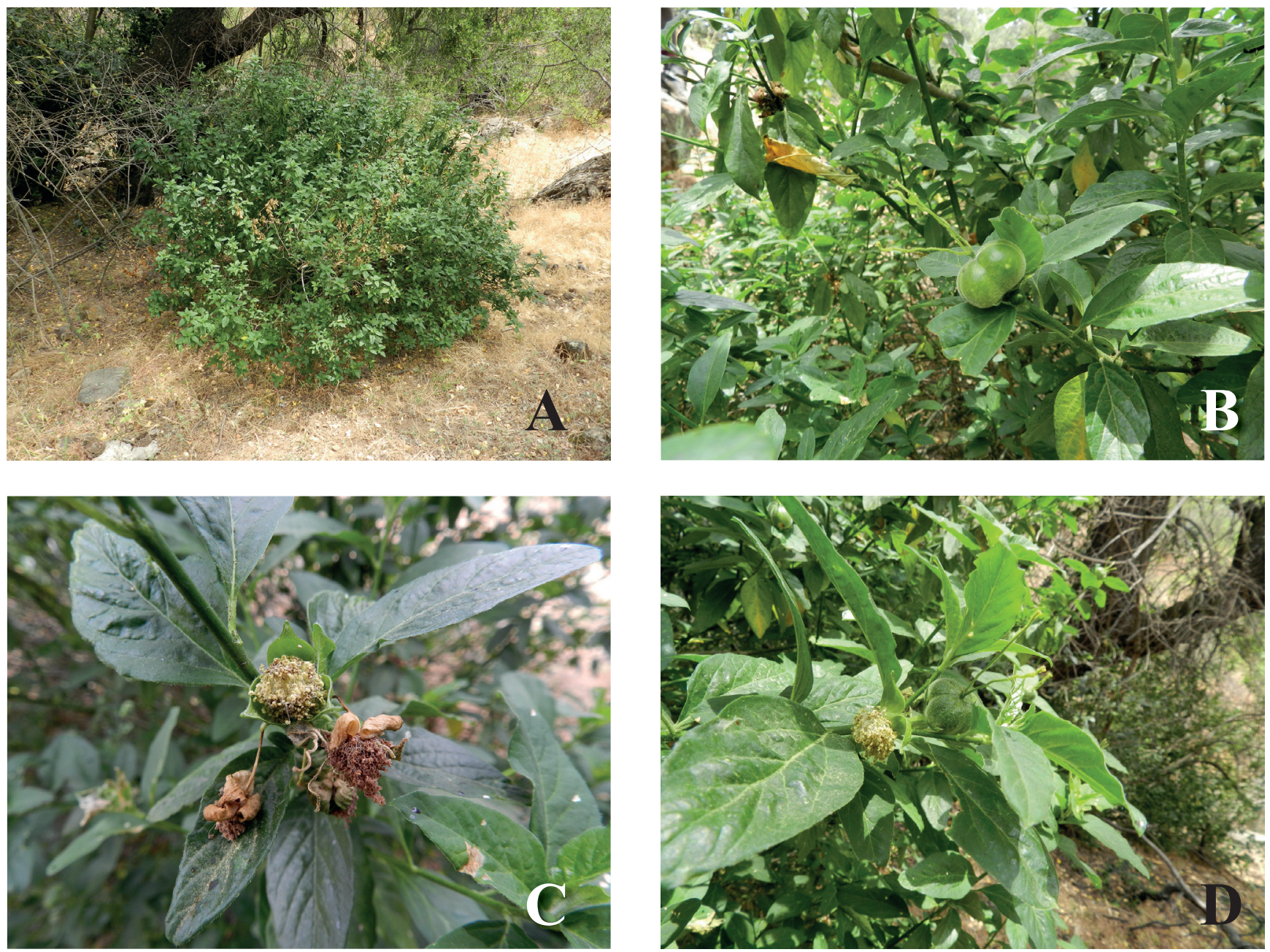

Figura 2: Individuo de Avellanita bustillosii registrado en fragmento de bosque esclerófilo en piedemonte del cerro Retamal. A= Hábito. $\mathrm{B}=$ Fruto en desarrollo. $\mathrm{C}=$ Flor masculina. $\mathrm{D}=$ Fruto y flor masculina. Noviembre 2013. / An individual of Avellanita bustillosii, recorded in a sclerophyllous forest fragment at base of Retamal hill. $\mathrm{A}=$ Habit. $\mathrm{B}=$ Developing fruit. $\mathrm{C}=$ Male flower. $\mathrm{D}=\mathrm{Male}$ flower and fruit. November 2013. 
argumenta la necesidad de reevaluar su categoría de estado de amenaza. Según los criterios empleados a nivel nacional (UICN 2012, Díaz-Páez et al. 2004, Squeo et al. 2010), se plantea la enmienda de la categoría actual En Peligro (EN) y Rara (R) (CONAMA 2006, Ricci \& Benoit 2008), por la categoría En Peligro Crítico (CR), de la misma manera que lo expuesto por Hechenleitner et al. (2005). Los argumentos para la proposición de la categoría indicada se basan en los siguientes aspectos: primero, la especie presenta un área de ocupación acotada y concentrada en tres localidades viables conocidas $\left(<10 \mathrm{~km}^{2}\right.$, Tabla 1). A pesar que la población de Laguna Aculeo comprende el área de ocupación más grande, abarca un mayor número y tamaño de fragmentos, sumado a que no existe claridad respecto al número total de parches existentes en la localidad ni un catastro de puntos de distribución. Segundo, todas las comunidades de bosque esclerófilo con presencia de avellanita se encuentran severamente fragmentadas, con escasos parches conocidos y con un patrón de distribución en el espacio aislado y en extremo localizado bajo condiciones de microhábitat particulares. Tercero, se plantea que las poblaciones conocidas poseen un nivel de fragilidad elevado y se encuentran próximas a la extinción, pues cualquier factor que altere la calidad de hábitat, las comunidades donde habita y genere la disminución de su tamaño poblacional, eventualmente podría afectar una población en su totalidad y en consecuencia la desaparición de una localidad dentro de su estrecho rango de distribución, dado que todas las localidades presentan numerosas amenazas de origen antrópico y son susceptibles a la estocasticidad ambiental propia de los ecosistemas mediterráneos de Chile Central. Por otra parte, se estimó la superficie de extensión de la presencia (Tabla 1). Sin embargo, gran parte de la superficie incluida comprende terrenos de cultivo, cumbres de cordones montañosos y condiciones ambientales que no conforman parte de los requerimientos de hábitat de la especie (laderas expuestas al norte, alta pendiente, entre otros), lo que permitiría señalar que la magnitud estimada sobrestima el valor real, sobre todo si se compara con el monto del área de ocupación calculado.

La magnitud de la estimación de extensión de la presencia definida en el presente trabajo para la especie, alcanza una superficie de $940 \mathrm{~km}^{2}$. El polígono generado tiene una forma alargada y angosta puesto que las localidades conocidas presentan un patrón de distribución alineado (Fig. 1). El área de ocupación para la especie alcanza 698,4 ha, cerca de $7 \mathrm{~km}^{2}$ (Tabla 1). Sobre la base de la extensión de la presencia, área de ocupación, número de individuos y amenazas imperantes, se generó una reevaluación de la categoría de estado de amenaza de acuerdo a criterios UICN

TABLA 1: Tamaño poblacional, área de ocupación y extensión de la presencia para el total y la nueva localidad descrita. *Tamaño poblacional corresponde a una magnitud estimada tras la revisión de Venegas y Contreras (2003), Ricci y Benoit (2008), aunque información proporcionada por Romero (2013, comm. pers.) indica un número mayor de individuos. ***Tamaño poblacional abarca 3 de 4 fragmentos descritos, no existe datos de número de individuos para fragmento D. / Demographic size, Area of Occupancy and extension of the presence for the total and new location recorded. * The demographic size it means an estimate magnitude_after_review Venegas \& Contreras (2003) and Ricci \& Benoit (2008), although information provided by F. Romero (comm. pers. 2013) indicates a larger number of individuals. $\stackrel{* *}{-}$ The demographic size provided includes 3 of 4 fragments described and there is no data about the D fragment in new record site.

\begin{tabular}{|c|c|c|c|}
\hline LOCALIDAD & Aculeo & Chancón & TROCALÁN y RETAMAL \\
\hline FuENTE & Diversos AUTORES* & MEdinA, 2010 & Presente estudio** \\
\hline Tamaño población ( $\mathrm{N}^{\circ}$ Individuos) & $2000_{-}^{*}$ & 6320 & $803 * *$ \\
\hline Tamaño población ( $\mathrm{N}^{\circ}$ Individuos) & \multicolumn{3}{|c|}{9123} \\
\hline Área ocupación parcial (ha/ km²) & $693,9 / 6,93$ & $2,90 / 0,03$ & $1,60 / 0,02$ \\
\hline Área ocupación $\left(\mathrm{km}^{2}\right)$ & \multicolumn{3}{|c|}{6,98} \\
\hline Extensión presencia parcial $\left(\mathrm{ha} / \mathrm{km}^{2}\right)$ & $4955,7 / 49,55$ & $11,4 / 0,11$ & $105,7 / 1,05$ \\
\hline Extensión presencia $\left(\mathrm{km}^{2}\right)$ & \multicolumn{3}{|c|}{940,71} \\
\hline Principales amenazas & $\begin{array}{l}\text { Incendios, cambio uso suelo, } \\
\text { urbanización, ramoneo }\end{array}$ & $\begin{array}{c}\text { Incendios, minería, } \\
\text { desmonte bosque nativo, } \\
\text { extracción tierra de hojas }\end{array}$ & $\begin{array}{c}\text { Minería, incendios, desmonte } \\
\text { bosque nativo }\end{array}$ \\
\hline Requerimientos & $\begin{array}{c}\text { Efectuar censo y } \\
\text { georeferenciación detallada de } \\
\text { fragmentos }\end{array}$ & $\begin{array}{l}\text { Revisita: monitoreo y } \\
\text { actualización información }\end{array}$ & $\begin{array}{c}\text { Censo fragmento } \mathrm{D} \text {, búsqueda } \\
\text { nuevos fragmentos }\end{array}$ \\
\hline
\end{tabular}


(2012), la cual modificaría la categoría definida en DS $\mathrm{N}^{\circ}$ 151/2007 al estatus En Peligro Crítico (CR), cumpliendo con los criterios B2ab (iii).

El presente hallazgo consiste en el primer registro de Avellanita bustillosii, en cordones montañosos de la vertiente oriental de la precordillera andina de Chile Central. Esta situación modifica en parte las hipótesis acerca de su historia natural, patrones de distribución geográfica, biogeografía y pone en duda el actual conocimiento acerca de la corología de la especie dentro de su área de extensión, dado que la información disponible a la fecha indicaba que la especie sólo se asociaba de manera única a bosques esclerófilos de la vertiente oriental de la Cordillera de la Costa. El registro permitió ampliar el límite sur en $26,5 \mathrm{~km}$, conformando la población conocida más austral. Además, se observó que corresponde a la población ubicada en comunidades de bosque esclerófilo de mayor elevación. No se descarta la posibilidad que el aumento de tiempo y esfuerzo de muestreo en la sección preandina de las provincias de Maipo y Cachapoal permitan detectar nuevos sitios de registro para la Región de Valparaíso, Metropolitana y la Región de O'Higgins.

Resulta lamentable, que las comunidades de bosque esclerófilo de la vertiente oriental de la Cordillera de la Costa donde habita la avellanita no posean representatividad en SNASPE (Hechenleitner et al. 2005, Ricci \& Benoit 2008, Medina 2010), sumado a que la totalidad de las poblaciones que conforman la corología del taxón se encuentran en predios de dominio privado (Ricci \& Benoit 2008, Medina 2010).

\section{AGRADECIMIENTOS}

A Alicia Marticorena por sus comentarios y al personal del Herbario del MNHN (SGO). A Larisa Donoso por el apoyo con software SIG y Figura 1. A todo el equipo que colaboró en la búsqueda de la avellanita (Fernanda Romero, Juan Rodríguez, Ximena Romero, entre otros).

\section{REFERENCIAS}

CONAF. 2017. Parques Nacionales; Parques de Chile. URL: http://www.conaf.cl/parques-nacionales/parques-de-chile/ Visita: 19 julio 2017.

CONAMA. 2004. Estrategia Conservación de la Biodiversidad en la Región Metropolitana de Santiago. 98 pp.

CONAMA. 2006. Primer Proceso de Clasificación de Especies Silvestres: Ficha Técnica Avellanita bustillosii. Phil. URL: http://www.conama.cl/clasificacionespecies/lista especies_primer_proceso.htm Visita: 7 junio 2017.

CONAMA. 2008. Estrategia Conservación de la Biodiversidad Región del Libertador Bernardo O’Higgins. 24 pp.
Díaz-Páez, H., Vidal, M., Berrios, P., Bocaz, P., González, P., Moreno, R., Murillo, W., Rodríguez, S., Sánchez, R., Villagrán-Mella, R., Ortíz, J., Úbeda, C. 2004. Revisión y Análisis de los Criterios y Métodos para la Categorización en Estados de Conservación de las Especies de Flora y Fauna en Chile. Boletín de la Sociedad de Biología de Chile 75: 73-89.

Hechenleitner, V. P., Gardner, M., Thomas, P., Echeverría, C., Escobar, B., Brownless, P., Martínez, C. 2005. Plantas Amenazadas del Centro-Sur de Chile: Distribución, Conservación y Propagación. Primera Edición. Universidad Austral de Chile y Real Jardín Botánico de Edimburgo. $188 \mathrm{pp}$.

Jaffuel, F., Pirion, A. 1921. Plantas Fanerógamas del Valle de Marga - Marga. Revista Chilena de Historia Natural 25: 350-405.

Medina, P. 2010. Caracterización ambiental y estructural de la población de Avellanita bustillosii Phil. (Euphorbiaceae), en la localidad de Lo Chancón, Región del Libertador Bernardo O'Higgins. Memoria Título Profesional. Ingeniería Forestal. Facultad Ciencias Forestales y Conservación de la Naturaleza, Universidad de Chile. Santiago, Chile. 87 pp.

Muñoz, D. 2009. Prospección de poblaciones de Avellanita bustillosii Phil. en la Región Metropolitana. Informe práctica profesional. Ingeniería Forestal. Facultad Ciencias Forestales y Conservación de la Naturaleza, Universidad de Chile. Santiago, Chile. CONAF. 33 pp.

PHILIPPI, R.A. 1895. Plantas nuevas chilenas de las familias que corresponden al tomo V de la obra de Gay: (continuación). Anales Universidad de Chile 91: 511-513

Ricci, M., BEnort, I. (eds.). 2008. Plan nacional de conservación de la Avellanita, Avellanita bustillosii Phil. en Chile. CONAF. $39 \mathrm{pp}$.

Serey, I., Ricci, M., Smith-Ramírez, C. (eds.). 2007. Libro Rojo de la Región de O'Higgins. CONAF -Universidad de Chile. 206 pp.

Serra, M.T., Luebert, F., Ricci, M. 2005. Nuevo Registro para Avellanita bustillosii Phil. (Euphorbiaceae). Noticiario Mensual del Museo Nacional de Historia Natural 355: 1115.

Squeo, F., Estades, C., Bahamondes, N., Cavieres, L., Rojas, G., Benoit, I., Parada, E., Fuentes, A., Avilés, R., Palma, A., Solís, R., Guerrero, S., Montenegro, G., Torres-Mura, J. 2010. Revisión de la clasificación de especies en categorías de amenaza en Chile. Revista Chilena de Historia Natural 83: 511-529.

UICN. 2012. Categorías y criterios de la lista roja de la UICN: Versión 3.1. Segunda Edición. Gland, Suiza y Cambridge, Reino Unido. 42 pp.

Venegas, A., Contreras, X.(eds.). 2003. Programa de Conservación de Avellanita bustillosii Phil. (Euphorbiaceae): Especie en Peligro de Extinción. SAG RM, CONAMA RM. Santiago, Chile. 82 pp.

Videla, J. 2008. Destrucción del hábitat de Avellanita bustillosii Phil. Informe CONAF, Región de O'Higgins, Rancagua. 4 pp.

Recibido: 25.04.2016

Aceptado: 13.09 .2017 